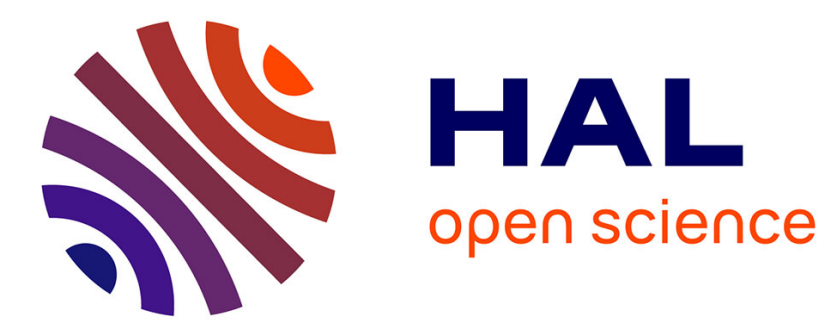

\title{
Six-dimensional view of dielectric mixtures as metamaterials
}

A. Sihvola

\section{To cite this version:}

A. Sihvola. Six-dimensional view of dielectric mixtures as metamaterials. European Physical Journal: Applied Physics, 2008, 46 (3), pp.1-5. 10.1051/epjap:2008104 . hal-00480176

\section{HAL Id: hal-00480176 https://hal.science/hal-00480176}

Submitted on 3 May 2010

HAL is a multi-disciplinary open access archive for the deposit and dissemination of scientific research documents, whether they are published or not. The documents may come from teaching and research institutions in France or abroad, or from public or private research centers.
L'archive ouverte pluridisciplinaire HAL, est destinée au dépôt et à la diffusion de documents scientifiques de niveau recherche, publiés ou non, émanant des établissements d'enseignement et de recherche français ou étrangers, des laboratoires publics ou privés. 


\title{
Six-dimensional view of dielectric mixtures as metamaterials
}

\author{
Ari Sihvola \\ Helsinki University of Technology \\ Department of Radio Science and Engineering \\ P.O. Box 3000, FI-02015 TKK, Finland
}

\begin{abstract}
This paper discusses homogenization formulas and mixing rules that connect effective dielectric properties of mixtures with their microstucture. The special point of view in this paper is that all parameters appearing in mixing formulas are treated with equal status. The parameters that can be varied are the permittivities (which are allowed to be negative as well as positive) of the inclusion, environment, and composite, the volume fraction, spatial dimension, and an abstract parameter which chooses the given mixing formula type. The analysis helps in the interpretation of predictions from classical mixing rules when they are extended into the domain of negative-permittivity metamaterials.
\end{abstract}

Submitted to EPJ Applied Physics

Special Issue on Metamaterials, guest-editors S. Zouhdi and A. Razek

April 10, 2008 


\section{Introduction}

For understanding natural materials and designing engineered composites, electromagnetic research has provided homogenization theories and mixing formulas that give estimates for the macroscopic electric and magnetic properties of these materials $[1,2,3]$. The classical use of mixing formulas has been to calculate the effective permittivity of a sample of heterogeneous medium, given the permittivies of the components and their fractional volumes in the mixture.

Methods to compute or even to estimate effective material parameters are needed in the remote sensing of natural environment where the dielectric response of geophysical media needs to be understood [4], in composite materials design [5], and especially within the emerging field of metamaterials research [6].

This presentation takes a new look at the homogenization problem, generalized in two respects. First, the restriction to the class of ordinary, conventional materials is relaxed, and the permittivities are allowed to reach also negative values as well as positive. Secondly, the usual way of looking at mixing rules as explicit formulas to calculate the effective permittivity when the properties of the mixture are given, is replaced by a multidimensional point of view. In other words, mixing rules are seen as connected relations where the parametrizations in all dimensions are placed on equal footing. The rank of this multidimensional world is six: these parameters are the three permittivities (environment phase, inclusion phase, effective medium), fractional volume, dimensionality of the space, and an abstract parameter $\nu$ connected to the type of mixing rule. On the other hand, in some other respects the degreees of freedom in the mixtures under consideration are restricted: there are only two component phases making the mixture, these materials are isotropic (their permittivity is equivalent to a scalar), and the underlying geometry is based on spherical building blocks.

\section{Mixing relations}

A great number of homogenization theories exist to explain the relation between the effective permittivity of a mixture $\epsilon_{\text {eff }}$ as a function of the inclusion permittivity $\epsilon_{i}$, environment permittivity $\epsilon_{e}$, and the inclusion volume fraction $f$. A generalized mixing formula which attempts to capture a large amount of these homogenization principles, for the case of isotropic spherical inclusions, reads [7]

$$
\frac{\epsilon_{\mathrm{eff}}-\epsilon_{e}}{\epsilon_{\mathrm{eff}}+2 \epsilon_{e}+\nu\left(\epsilon_{\mathrm{eff}}-\epsilon_{e}\right)}=f \frac{\epsilon_{i}-\epsilon_{e}}{\epsilon_{i}+2 \epsilon_{e}+\nu\left(\epsilon_{\mathrm{eff}}-\epsilon_{e}\right)}, \quad \text { in } 3 \text { dimensions }
$$

In this formula, the role of the unitless parameter $\nu$ is to pick various mixing rules which are known in the literature. Different choices of $\nu$ recover the following results: $\nu=0$ gives the Maxwell Garnett rule [8], $\nu=2$ gives the Bruggeman (symmetric) formula 
[9], and $\nu=3$ gives the Coherent potential mixing rule [10]. It is obvious that since the geometry of the mixture (spherical inclusions, random arrangement) and the component material responses are isotropic, also the effective continuum is isotropic and $\epsilon_{\text {eff }}$ is scalar.

This relation $(1)$ applies for three-dimensional $(d=3)$ spheres. Generalized into any spatial dimension $d$, the formula looks like

$$
\frac{\epsilon_{\mathrm{eff}}-\epsilon_{e}}{\epsilon_{\mathrm{eff}}+(d-1) \epsilon_{e}+\nu\left(\epsilon_{\mathrm{eff}}-\epsilon_{e}\right)}=f \frac{\epsilon_{i}-\epsilon_{e}}{\epsilon_{i}+(d-1) \epsilon_{e}+\nu\left(\epsilon_{\mathrm{eff}}-\epsilon_{e}\right)}, \quad \text { in } d \text { dimensions }
$$

Here the number of parameters has grown to six: the quantities are $\epsilon_{\mathrm{eff}}, \epsilon_{i}, \epsilon_{e}, f, \nu, d$.

The appearance of Equation (2) is certainly different from the way the well-known mixing formulas like Maxwell Garnett or Bruggeman are normally given. ${ }^{1}$ To calculate the effective permittivity from this equation, a second-order equation needs be solved. The physically acceptable solution for this second-order equation has to be chosen with the correct sign of the imaginary part of $\epsilon_{\mathrm{eff}}^{\prime}-\mathrm{j} \epsilon_{\mathrm{eff}}^{\prime \prime}$ if the constituents are passive $\left(\epsilon_{e}^{\prime \prime} \geq 0, \epsilon_{i}^{\prime \prime} \geq 0\right)$, the effective medium cannot be active ( $\epsilon_{\text {eff }}^{\prime \prime}$ has to be positive) ${ }^{2}$

The only case when no roots are necessary in the explicit expression for $\epsilon_{\text {eff }}$ is when $\nu=0$ which is the Maxwell Garnett formula. The solution with $\nu=0$ reads

$$
\epsilon_{\mathrm{eff}}=\epsilon_{e}+d f \epsilon_{e} \frac{\epsilon_{i}-\epsilon_{e}}{\epsilon_{i}+(d-1) \epsilon_{e}-f\left(\epsilon_{i}-\epsilon_{e}\right)}
$$

The advantage of the equation (2) is that it can be solved for any of the six parameters. As mentioned, the solution for $\epsilon_{\text {eff }}$ (except in the Maxwell Garnett case) leads to a second-order equation. The situation is the same when $\epsilon_{e}$ is solved. But for the other four parameters $\left(\epsilon_{i}, f, d, \nu\right)$, the solution is a first-order equation. This fact helps sometimes in computations for the effective permittivity when instead of directly solving for $\epsilon_{\text {eff }}$ one may solve for $f$. The resulting $\left(\epsilon_{\mathrm{eff}}-f\right)$-connection can be found out by varying $\epsilon_{\mathrm{eff}}$ between $\epsilon_{e}$ and $\epsilon_{i}$ and writing down the corresponding $f{ }^{3}$

It is true that with present-day computational methods to solve relatively simple equations like (2), such procedures to facilitate algebraic operations are not as essential as in earlier times. However, the idea of multidimensionality in a single formula and the possibility to view it from each parameter's point of view helps to glean physical interpretations of dielectric mixture behavior.

\footnotetext{
${ }^{1}$ Note, however, that the choice for Bruggeman formula depends on dimension: (for example, Bruggeman corresponds to $\nu=2$ for $d=3$, and to $\nu=1$ for $d=2$ ).

${ }^{2}$ Here the notation $\exp (\mathrm{j} \omega t)$ is taken for the time-harmonic field excitation.

${ }^{3}$ Of course such a procedure returns the whole range of values $0 \leq f \leq 1$ only in case of real permittivities, and with the assumption that $\epsilon_{\mathrm{eff}}$ always lies between the environment and inclusion permittivities which unfortunately is not always the case [11].
} 


\section{Application of the six-dimensionality}

Indeed, the connections in Equation (2) offer many possibilities to study the characteristics of various mixing rules. In this section, the multidimensionality is applied to illustrate some interesting phenomena in the response of dielectric materials.

\subsection{Percolation}

One of such interesting examples is the phenomenon of percolation $[12,13,14,15]$. In particular, percolation appears in different mixing rules in very varying ways. Percolation is a nonlinear phenomenon in the sense that a very abrupt change occurs in the macroscopic behavior. The geometry of the matter where percolation takes place is special: even with small changes in the fractions of the components forming the material, the structure behaves totally differently.

To see how the threshold of percolation comes from the mixing formula, let the dielectric contrast $\epsilon_{i} / \epsilon_{e}$ in Equation (2) become large. In that case, the equation is approximately

$$
\frac{\epsilon_{\mathrm{eff}}-\epsilon_{e}}{\epsilon_{\mathrm{eff}}+(d-1) \epsilon_{e}+\nu\left(\epsilon_{\mathrm{eff}}-\epsilon_{e}\right)}=f
$$

This gives the following expression for $\epsilon_{\mathrm{eff}}$ :

$$
\epsilon_{\mathrm{eff}}=\epsilon_{e} \frac{1+f(d-1-\nu)}{1-f(1+\nu)}
$$

However, this relation is only valid as long as $\epsilon_{\text {eff }} / \epsilon_{e}$ is small, i.e. only up to those volume fraction values where percolation appears. Clearly this result breaks down as the denominator reaches the value zero. That very point is interpreted as the percolation threshold point

$$
f=f_{c}=\frac{1}{1+\nu}
$$

Depending on $\nu$, the threshold varies between $f_{c}=1$ (Maxwell Garnett) and $f_{c}=0$ $(\nu=\infty)$. The well-known result of the percolation threshold of 0.333 for the Bruggeman symmetric formula is a simple corollary. Another noteworthy fact is that the threshold does not depend at all on the dimension of the space $(d) .{ }^{4}$

A fine-tuned approach to percolating mixtures can be followed using the generalized mixing formula by McLachlan and others [16]. The result (6), however, gives a straightforward connection between the mixing rule and percolation threshold.

\footnotetext{
${ }^{4}$ Because for the Bruggeman formula in two dimensions corresponds to $\nu=1$, the permittivity threshold is then $50 \%$ instead of $33 \%$.
} 


\subsection{Divergences of the effective permittivity}

The recipe (2) is especially powerful in the case of metamaterials when the restriction on the permittivity to be positive is relaxed. Then the predictions for the macroscopic response are very strongly dependent on $\nu$, in other words vary greatly with respect to different mixing rules. Singularities may appear, and complex effective permittivities may arise from real-valued components. Relation (2) can be solved for any of the six parameters, and the conditions for, for example, the singularities, can be calculated.

The solutions of (2) for $\epsilon_{\text {eff }}$ read

$$
\begin{aligned}
\epsilon_{\mathrm{eff}} & =\frac{A \pm \sqrt{A^{2}-4 \epsilon_{e} \nu B}}{2 \nu} \\
A & =\epsilon_{i}[f(1+\nu)-1]-\epsilon_{e}[d-1-2 \nu+f(1+\nu)] \\
B & =\epsilon_{i}[f(1-d+\nu)-1]+\epsilon_{e}(1-f)(1-d+\nu)
\end{aligned}
$$

This shows that there always exists a finite solution for $\epsilon_{\mathrm{eff}}$ for finite $\epsilon_{e}, \epsilon_{i}, d, f, \nu$ if only $\nu \neq 0 .{ }^{5}$ The problematic case is the Maxwell Garnett formula which corresponds exactly to $\nu=0$, and is hence the only member of the mixing family which can predict divergences.

Let us take a closer look at the Maxwell Garnett infinities for $\epsilon_{\text {eff }}$. The divergence happens exactly when the dimension of the space is

$$
d=(1-f) \frac{\epsilon_{e}-\epsilon_{i}}{\epsilon_{e}}
$$

To have an infinity for $\epsilon_{\text {eff }}$ requires of course that $\epsilon_{i}$ and $\epsilon_{e}$ have different signs, since otherwise condition (10) is not satisfied for any $d \geq 1$ and $0 \leq f \leq 1$.

Furthermore, the divergence requirement is stricter for higher densities. In other words, the larger $f$ is, the higher the negative contrast $\epsilon_{i} / \epsilon_{e}$ needs to be to cause an infinity in $\epsilon_{\text {eff }}$.

\subsection{Mixtures of positive and negative permittivities}

As a further example of peculiar predictions of various mixing formulas, let us analyze a mixture where the environment has a negative (relative) permittivity of $\epsilon_{e}=-1$, and the inclusions are air $\left(\epsilon_{i}=+1\right)$. Let $d=3$ which corresponds to three-dimensional spheres as inclusions. Figures 1 and 2 display the predictions of the various models for $\epsilon_{\text {eff }}$ of this mixture.

The figures show very interestingly the differing character of the various mixing rules. This variation is not obvious for "ordinary" mixtures with all the components having

\footnotetext{
${ }^{5}$ Sometimes the solution may be complex for real input parameters, due to the square root.
} 


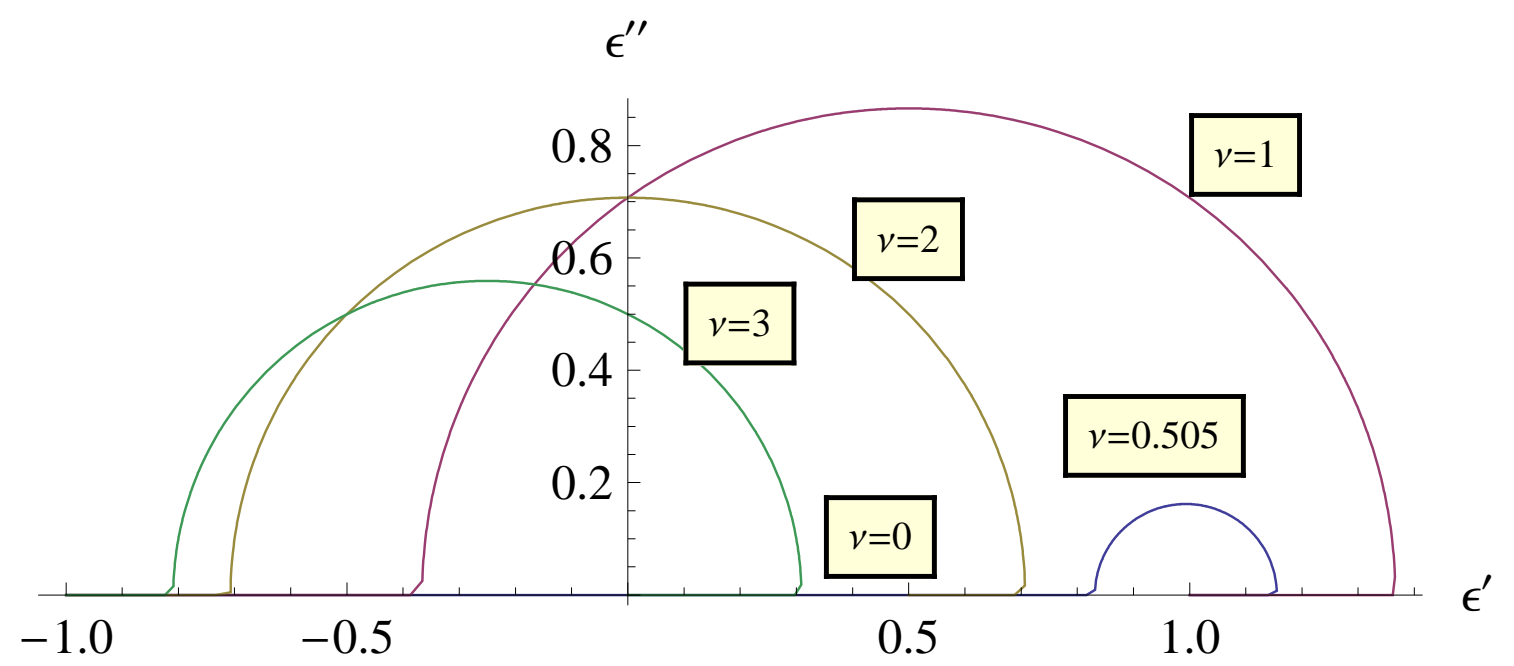

Figure 1: The effective permittivity in the complex plane for a mixture where the background permittivity is $\epsilon_{e}=-1$ and the inclusion permittivity $\epsilon_{i}=+1$, as the volume fraction goes from 0 to 1 . The predictions of the different mixing rules differ considerably: Maxwell Garnett $(\nu=0)$ does not predict any losses, and for formulas with $\nu$ exceeding the values 0.5 , the effective permittivity starts to display an imaginary part for a certain mixing ratio.
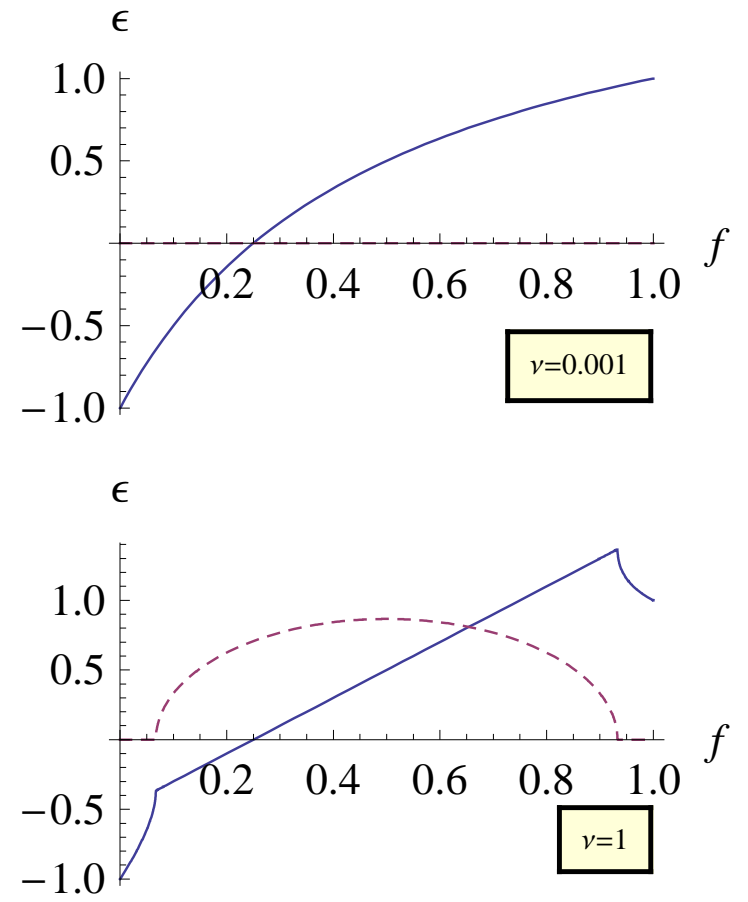
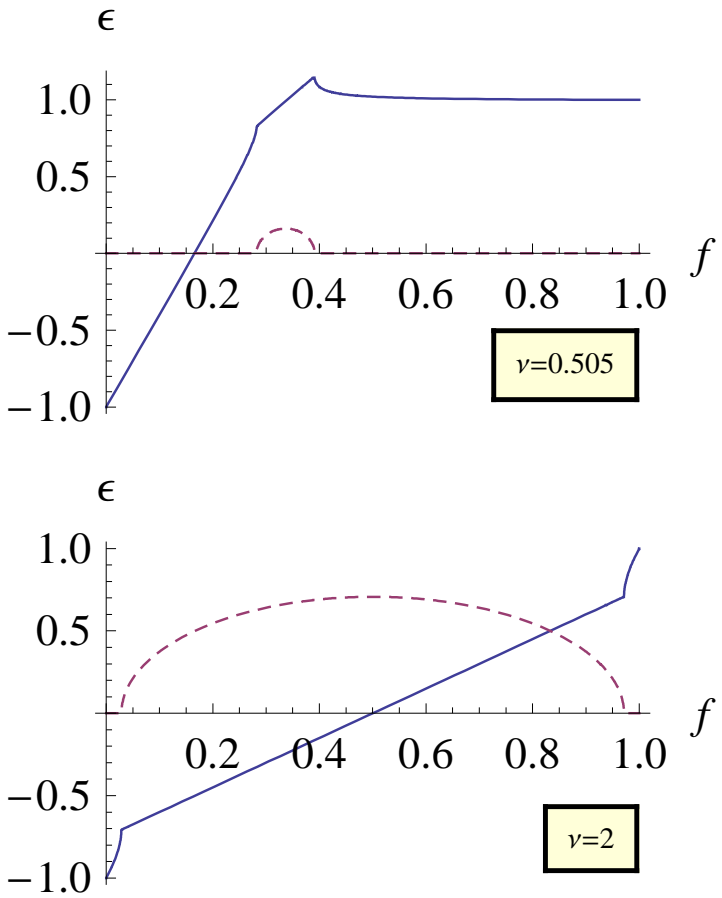

Figure 2: The same as in Figure 1 for the real (solid) and imaginary (dashed) parts of the effective permittivity as functions of the volume fraction $f$. 
positive permittivities. The most striking observation is that even if in the calculated example both phases composing the mixture were real, many of mixing rules predict that the composite have complex effective permittivity. It has been pointed out earlier in the literature that the Bruggeman formalism predicts lossy continuum for nondissipative component media [17], along with a strong conclusion that the Bruggeman formalism is not physically applicable for two-component isotropic mixtures.

Another observation concerning the Bruggeman prediction $(\nu=2)$ for the effective permittivity is the symmetry of the curve. The mixture under consideration is special $\left(\epsilon_{i}=-\epsilon_{e}\right)$, which leads to the fact that the 50-50 mixture $(f=0.5)$ gives $\epsilon_{\text {eff }}^{\prime}=0$, and the imaginary part has its maximum. The equal treatment of the host and guest materials in Bruggeman homogenization is a well-appreciated fact in the modeling of mixtures with positive-permittivity components.

Figures 1 and 2 cover a wider range of mixing rules with respsct to this appearance of dissipation in homogenization. It is obvious that Bruggeman formula predicts losses for the present mixture under study. However, the figures show that Bruggeman is not the homogenization principle giving the highest amplitude in losses: the case $\nu=1$ has stronger losses (and numerically seems to be the lossiest of all mixing rules for this mixture). Furthermore, there is a range of mixing rules (in the framework of the classification of Equation (1)) which behave like Maxwell Garnett formula and predict $\epsilon_{\text {eff }}^{\prime \prime}=0$ for all possible volume fractions $0 \leq f \leq 1$. This is the case for all mixing principles with $\nu<1 / 2$. Figures 1 and 2 give support to this as far as the present mixture $\epsilon_{i}=-\epsilon_{e}=1$ is concerned but it turns out to be valid also for any other values $\epsilon_{i}=-\epsilon_{e}{ }^{6}$

In Figure 3, the limits are mapped for which parameter values a given mixing rule predicts a non-zero imaginary part for the effective permittivity. This describes the case when the relative permittivity of the inclusions is $\epsilon_{i}=+1$ (like in Figures 1 and 2). The shaded area gives the background permittivity values for given volume fraction $f$ for which $\epsilon_{\text {eff }}$ will become complex.

As can be seen, the increase in $\nu$ has the effect that the shaded area increases. The Maxwell Garnett limit of vanishing $\nu$ can be seen as a zero-thickness complexity-causing area in the $\left(f, \epsilon_{e}\right)$ plane. But the cost of the vanishing area is that the effective permittivity is predicted to be infinitely large. For Maxwell Garnett, the area shrinks to a curve which matches exactly the divergence expression (10).

The complementary case is shown in Figure 4. There the corresponding map as in Figure 3 is drawn for the mixture where negative permittivity 3D spheres occupy a volume fraction $f$ in air $\left(\epsilon_{e}=+1\right)$. In other words, the role of the environment and inclusions are interchanged between Figures 3 and 4 . The area where $\epsilon_{\text {eff }}$ becomes complex is again shaded. Also here, the increase of $\nu$ will make the shaded area larger. It is worth noting the behavior of the complexity regimes of the small- $\nu$ predictions: the $f=1$ limit goes

\footnotetext{
${ }^{6}$ Also for negative values of $\nu, \epsilon_{\text {eff }}$ seems to remain real-valued. However, a strong conclusion about this claim would require further systematic study.
} 

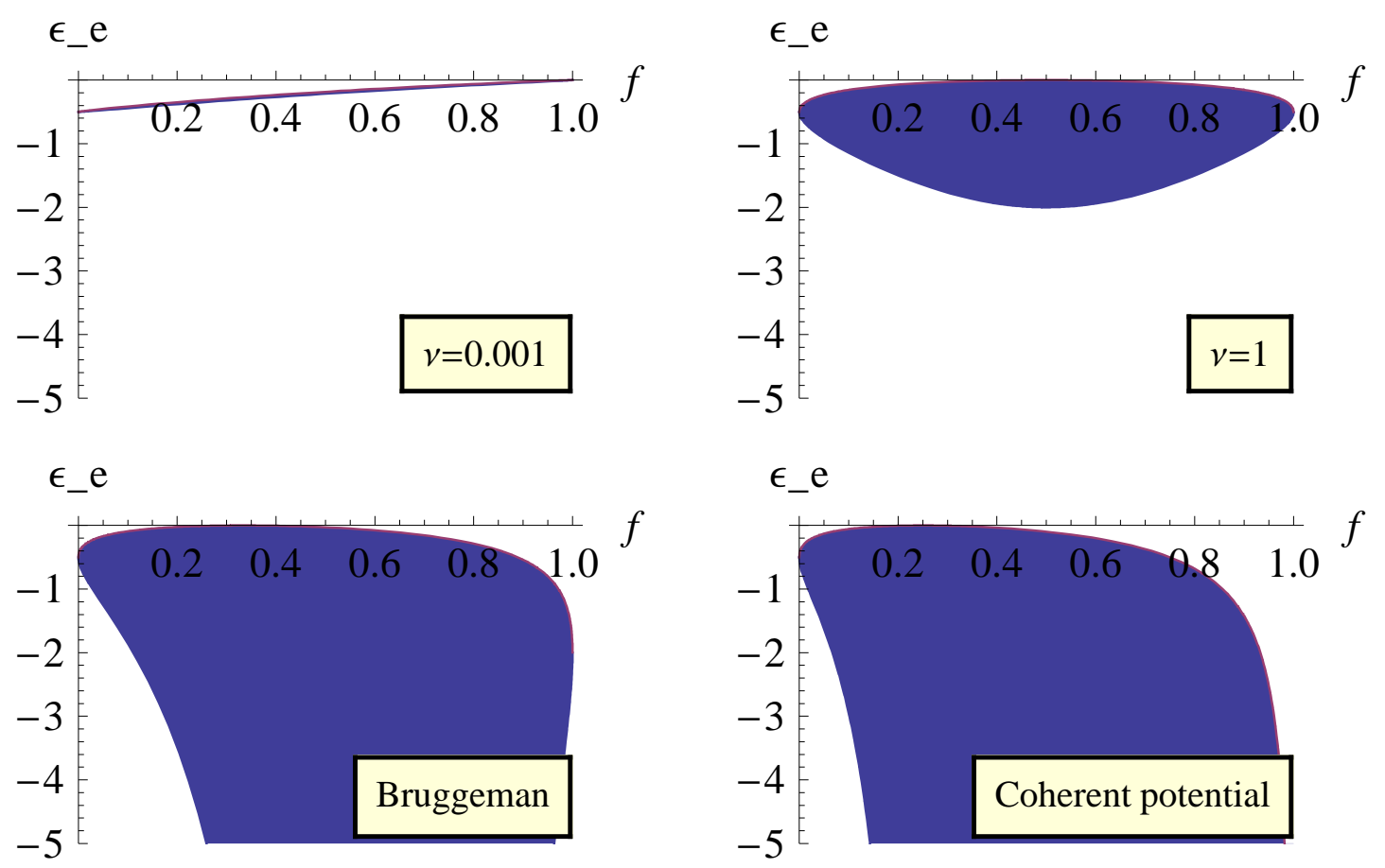

Figure 3: A map for emergence of complex effective permittivity for a mixture where a negative continuum is pierced by three-dimensional spherical holes (with relative permittivity $\epsilon_{i}=+1$ ). The shaded area gives the range values of the background permittivity $\epsilon_{e}$ and the volume fraction for which a non-zero imaginary part appears in $\epsilon_{\mathrm{eff}}$ for different mixing rules, characterized with the parameter $\nu$.

to $\epsilon_{i}=0$, whereas in the complementary case the negative-permittivity component was forced to go towards negative infinity.

Another peculiarity in Figure 4 is the behavior of the Coherent potential mixing formula $(\nu=3)$. Figure 4 shows that for some volume fraction values, the shaded area extends to positive values (although very small values) of the inclusion permittivity. This is a characteristic that is absent from the complementary mixture, as is seen in Figure 3.

\section{Conclusion}

The six-dimensional description of the prediction of the effective permittivity of mixtures offers new possibilities to describe and analyze macroscopic properties of heterogeneous media. Especially in the case of metamaterial mixtures where the permittivity is allowed to extend into negative values, the descriptions (1) and (2) can simplify the description of emergent phenomena and even provide new insights into the properties of metamaterials.

It is true that when the possibility of negative permittivities is combined with the com- 

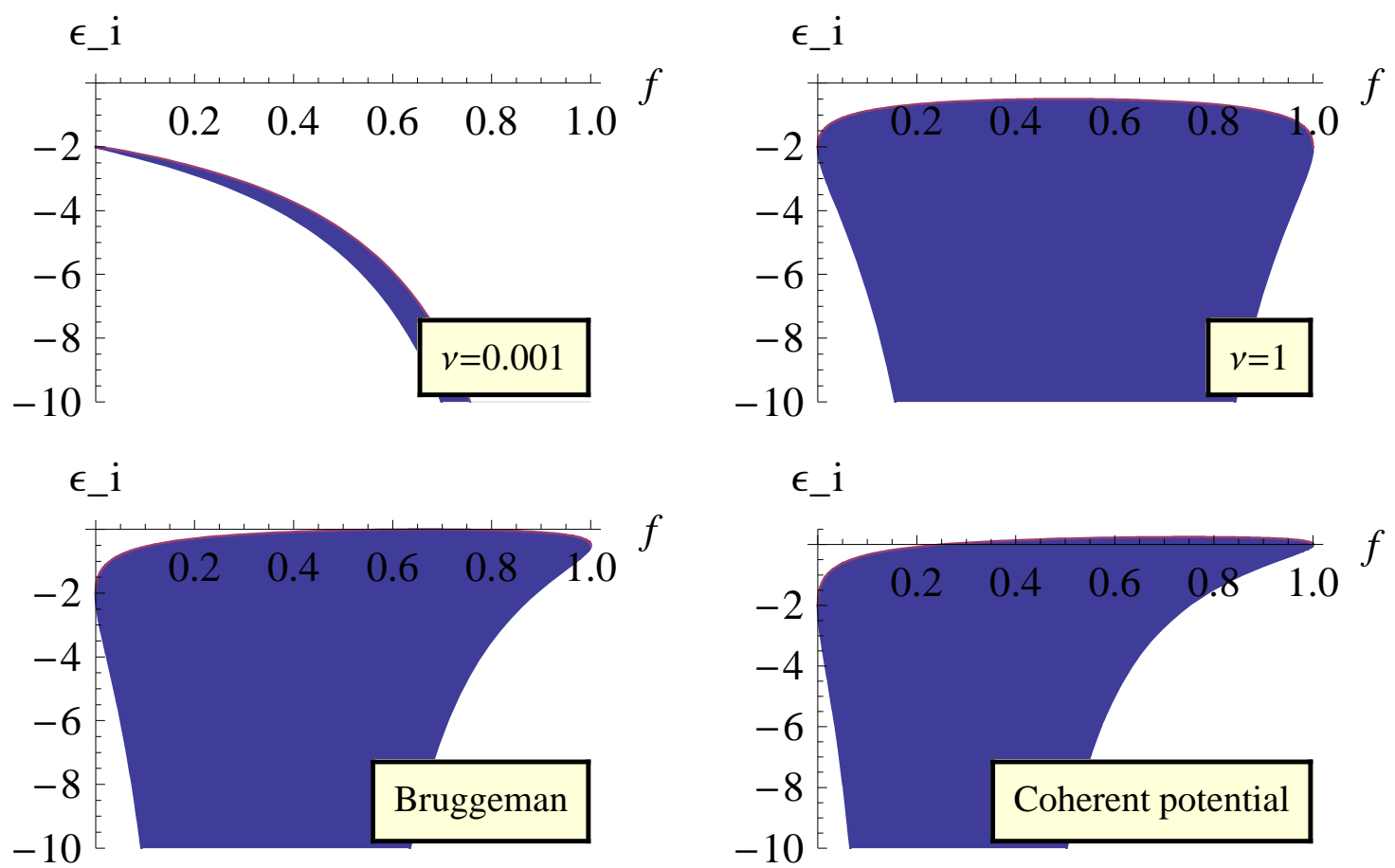

Figure 4: A similar map as in Figure 3, for the complementary mixture: the background permittivity is air $\left(\epsilon_{e}=+1\right)$ and the inclusion permittivity varies in the negative regime.

plications of mixing effects, sometimes counterintuitive predictions can be encountered, such as singularities, infinities, and complex-valued response parameters. For example Bruggeman mixing rule has been criticized for such behavior. It may be, however, a too strict conclusion to throw away the Bruggeman mixing rule for this type of positivenegative permittivity mixtures, only because of the dissipation that is created in the macroscopic permittivity. Maxwell Garnett rule as an alternative is also problematic with infinities for the continuum permittivity, which hurts everyday expectations. And these infinities are absent in mixing rules with $\nu \neq 0$, like Bruggeman mixing.

Another reason to tolerate mixing rules with complexity-predicting properties is that, after all, mixtures like the ones considered in Figures 1 and 2 are in the domain of metamaterials, having unconventional material parameters. And it is the very ethos of metamaterials that emergent properties appear in the higher-level homogenization. So why not accept emergent dissipation?

As a final reminder, it is important to keep in mind the limitation to spherical geometry of the analysis. Mixing formulas have been written also for ellipsoidal inclusion shapes [7]. However, to account for such geometries would require additional parameters into Equation (2). 


\section{References}

[1] R. Landauer, Electrical conductivity in inhomogeneous media, in Electrical transport and optical properties of inhomogeneous media, American Institute of Physics, Conference Proc. 40, 1978, edited by J.C. Garland and D.B. Tanner, pp. 2-45.

[2] P.S. Neelakanta, Handbook of electromagnetic materials, (CRC Press, Boca Raton, Florida, 1995)

[3] G.W. Milton, The theory of composites, (Cambridge University Press, 2002)

[4] F.T. Ulaby, R.K. Moore, and A.K. Fung, Microwave remote sensing, Active and passive, (Addison Wesley, Reading, Massachusetts, 1981 (Vol. 1), 1982 (Vol. 2); Artech House, Norwood, Massachusetts, 1986 (Vol. 3))

[5] A. Lakhtakia (editor), Selected papers on linear optical composite materials, (SPIE Optical Engineering Press, Bellingham, Washington, 1996)

[6] N. Engheta and R.W. Ziolkowski (editors), Metamaterials: physics and engineering explorations, (John Wiley \& Sons, 2006)

[7] A. Sihvola, Electromagnetic mixing formulas and applications, (IEE Press, London, 1999)

[8] J.C. Maxwell Garnett, Colours in metal glasses and in metal films, Transactions of the Royal Society of London, Vol. 203, 1904, pp. 385-420

[9] D.A.G. Bruggeman, Berechnung verschiedener physikalischer Konstanten von heterogenen Substanzen, I. Dielektrizitätskonstanten und Leitfähigkeiten der Mischkörper aus isotropen Substanzen, Annalen der Physik, 1935, Series 5, 24, pp. 636-679

[10] W.E. Kohler and G.C. Papanicolaou, Some applications of the coherent potential approximation, in P.L. Chow, W.E. Kohler, and G.C. Papanicolaou (Eds.), Multiple scattering and waves, (North Holland, New York, 1981), pp. 199-223

[11] A. Sihvola, How strict are theoretical bounds for dielectric properties of mixtures? IEEE Transactions on Geoscience and Remote Sensing, 40(4), 880-886, April 2002.

[12] G. Deutscher, R. Zallen and J. Adler (editors), Percolation processes and structures, Annals of the Israel Physical Society, Vol. 5, (Adam Hilger, Bristol, 1983)

[13] G. Grimmett, Percolation, (Springer, New York, 1989)

[14] D.S. McLachlan, M. Blaszkiewicz and R.E. Newnham, Electrical resistivity of composites, Journal of the American Ceramic Society, 73(8), 2187-2203, 1990.

[15] D. Stauffer and A. Aharony, Introduction to percolation theory, Revised Second Edition, (Taylor and Francis, London, 1994) 
[16] D.S. McLachlan, A. Priou, I. Chenerie, E. Issac and F. Henry, Modeling the permittivity of composite materials with a general effective permittivity equation, Journal of Electromagnetic Waves and Applications, 6(9), 1099-1131, 1992.

[17] T.G. Mackay and A. Lakhtakia, A limitation of the Bruggeman formalism for homogenization, Optics Communications, Vol. 234, pp. 35-42, 2004 